\title{
A Flow Cytometric and Cytochemistric Analysis of Urine to Detect Early Urinary Tract Infection
}

\author{
Mona Bargotya ${ }^{1}$, Lalit Kumar ${ }^{1 *}$, Pinkey Kachhap ${ }^{1}$, Payel Das ${ }^{1}$, Vidushi Sachdeva ${ }^{1}$, Sonali Bhattar ${ }^{2}$ \\ 'Department of Pathology, Rajiv Gandhi Super Speciality Hospital, New Delhi, India \\ ${ }^{2}$ Department of Microbiology, Rajiv Gandhi Super Speciality Hospital, New Delhi, India
}

\begin{abstract}
Introduction: Urinary tract infections (UTI) are infections affecting any part of the urinary tract and are most commonly caused by a multitude of bacteria. Most tests used these days to detect UTI are based on chemical test strips and cultures, both of which are labor and time-intensive. The aim of our study is to establish parameters that could detect UTI with high certainty.

Material \& Methods: The study was conducted in the Department of Pathology of Rajiv Gandhi Super Speciality Hospital, Delhi. The samples received between January to March 2017 were included in the study. All the samples were run in the Sysmex UX 2000 , a fully automated urine analyzer.

Results: A total of 236 patients were registered in this study. The study participants range from 4 years to 88 years with $56 \%$ males and $44 \%$ females. Out of these $21 \%$ were positive for UTI whereas $9 \%$ were inconclusive and $70 \%$ were negative. Cutoff values for white blood cells (WBC) and bacterial counts taken were 20.2 cells $/ \mu 1$ and 143.2 cells/ $\mu 1$, respectively. Flowcytometric analysis of both WBC and bacterial cells showed high sensitivity (81\%) as well as high negative predictive value (NPV) of $93 \%$. Analysis of nitrites showed $97 \%$ specificity and $81 \%$ NPV, while leucocyte esterase showed $87 \%$ specificity and $92 \%$ NPV.
\end{abstract}

Conclusion: Flow cytometry-based bacterial counts, total leucocyte counts, as well as nitrites and leucocyte esterase, could be used as screening tests for early as well as subclinical urinary tract infections and will reduce the need for urine culture.

\section{Keywords: Urinary Tract Infection, Flow Cytometry, Leucocyte Esterase, Nitrites, Bacterial Counts}

\section{Introduction}

Urinary tract infections (UTI) are one of the most common causes of infections. It accounts for $25 \%$ of all infections and affects 150 million people per year worldwide. It can occur in any population and age groups, however, is most common in females in reproductive age..$^{[1,2]}$ UTI is a term that is used to describe a group of diseases like acute uncomplicated bacterial pyelonephritis, recurrent cystitis and asymptomatic bacteriuria. ${ }^{[1,3]}$ Uncomplicated UTI is more commonly seen in the female population while the men suffer more from complicated UTI. ${ }^{[4]}$ Incidence of UTI increases with increasing age. The patients generally present with dysuria, increased frequency of micturition, hematuria and back pain. ${ }^{[5]}$

Urinary tract infections (UTI) are infections which can affect any part of the urinary tract and are most commonly caused by a multitude of bacteria. E.coli is responsible for more than $95 \%$ cases of urinary tract infections. Other organisms causing UTI's are Klebsiella, Staphylococcus, Enterobacter, Proteus, Pseudomonas and Enterococci species. These are more commonly isolated from inpatients..$^{[4,6]}$
Manual urinalysis is commonly done by chemical strips that can detect White Blood Cells (WBC), Red Blood Cells (RBC), $\mathrm{pH}$, nitrite and leucocyte esterase. For the determination of cells, casts, crystals and bacteria, microscopic analysis of the urine sediment is done. Manual urinalysis using dipstick and sediment analysis is a good screening test. ${ }^{[7-10]}$ Confirmation of UTI is done by culture, which is considered the gold standard. ${ }^{[11]}$ Urine culture adds to the workload of the laboratory as it takes 18 - 48 hours, is an expensive test and delays the initiation of appropriate treatment. ${ }^{[12,13]}$

Flow cytometry-based automated urine analyzers are in use nowadays which reduces the urine screening time to mere minutes. The Sysmex UX 2000 is an automated urine flow cytometry and cytochemistry based analyzer which can detect particles in urine like WBC, RBC, casts, crystals and bacteria and at the same time use chemical strips to detect the concentration of glucose, proteins, nitrites and leucocyte esterase. ${ }^{[14]}$ It can be used as a rapid screening test for the detection of UTI.

The aim of the study is to determine chemical as well as flow cytometry based parameters using Sysmex UX 2000 which would aid in early detection of UTI. 


\section{Materials \& Methods}

The study was conducted in the Department of Pathology of Rajiv Gandhi Super Speciality Hospital. All the midstream urine samples collected in a sterile container received between January 2017 to March 2017 and submitted in the Department of Pathology were enrolled in the study. Samples not collected by mid-stream method or collected in a non-sterile container were excluded from the study.

Each sample underwent biochemical and flow cytometric examination using the Sysmex UX 2000 (Sysmex, Japan), a fully automated urine analyzer. The UX 2000 aspirates $2.2 \mathrm{ml}$ of urine; $0.95 \mathrm{ml}$ for cytochemistry (CHM) and $1.2 \mathrm{ml}$ for flow cytometry (FCM). It uses transmission refractometry to detect specific gravity, light scattering for determining turbidity and reflectivity for color detection. Dual-wavelength reflectance method is used by CHM for the detection of leucocyte esterase and nitrite. For flow cytometry, the aspirated sample is stained with a fluorescent dye and analyzed in two channels; one for WBC and other for bacteria. The sample passes through the flow channel where the laser beam strikes each particle individually and produces forward scattered light signal, laterally scattered light signal and lateral fluorescent light signal that is detected and converted into electrical signals. All signals are analyzed as scattergrams. This categorizes the particles in urine into RBC, WBC, epithelial cells, cast and bacteria using a classification algorithm. ${ }^{[14,15]}$ The urine analyzer undergoes strict quality control checks for both CHM and FCM every day. In this study, we evaluated four parameters, namely $\mathrm{WBC}$ counts, bacterial counts (BAC), leucocyte esterase (Leu) and nitrites (Nit), in all the specimens.

All the samples were reexamined with the dipstick method using strips which can detect $\mathrm{pH}$, specific gravity, leucocyte esterase, nitrites, glucose, proteins, ketones, bilirubin and urobilinogen. Microscopic sediment analysis was performed on each urine sample. The sample was centrifuged at $1000 \mathrm{rpm}$ for $10 \mathrm{~min}$, the supernatant was removed, the pellet was suspended and a drop is put on a glass slide and cover-slipped. The prepared slides were examined at 400x (HPF). For each sample, at least 20 HPFs were examined.

Also, each urine sample was cultured within half an hour of receiving the sample. The samples were inoculated on Mac Conkey agar as well as blood agar and were incubated for 24 hours at $37^{\circ}$. In negative cases, the samples were incubated up to 48 hours. The culture was considered positive when it yields $\geq 10^{5} \mathrm{CFU} / \mathrm{ml}$. Gram stain and recommended biochemical tests were done for bacterial identification. In the presence of more than two species without a dominant species, the sample was considered as contaminated and rejected. ${ }^{[16]}$

The statistical analysis was performed using Microsoft Excel (Microsoft Corporation, Washington, USA) as well as SPSS statistical program version 25 (IBM, Chicago, USA).

\section{Results}

Total 236 patients were registered in the present study with age ranging from 4 years to 88 years. Out of 236 registered patients, $133(56.4 \%)$ patients were male and $103(43.6 \%)$ were females. The mean age for men was $47.4 \pm 17.7$ years and $41.2 \pm 14.7$ years for the women. Out of the 236 samples received, 49 (20.8\%) came culture positive, i.e. the culture yielded $\geq 10^{5} \mathrm{CFU} / \mathrm{ml}, 166$ (70.3\%) samples were negative and the rest $21(8.9 \%)$ samples were inconclusive. The majority of urine samples $(78.3 \%)$ received from men in the study turned out to negative, while $60 \%$ of samples from female patients were negative.

The most common organism identified was E. coli $(70 \%)$ followed by Enterobacter faecalis (11\%), Klebsiella, Staphylococcus sp. and Candida sp. One case of Staphylococcus showed no significant changes on routine screening. Four parameters were compared, taking culture results as the gold standard, for establishing an early diagnosis of UTI, which includes WBC, BAC, leucocyte esterase and nitrites. Mean WBC counts were 881.9 cells $/ \mu 1$ for culture positive samples and 107.5 cells $/ \mu 1$ for negative. Mean BAC counts were 9619.8 cells/ $\mu 1$ for culture positive samples and 160.3 cells/ $\mu 1$ for negative. Receiver operating curve was plotted to establish the cut off values for $\mathrm{WBC}$ and $\mathrm{BAC}$ parameters. The area under the curve (AUC) for WBC was 0.87 (95\% CI, 0.81-0.92; $\mathrm{p}<0.0001)$ and $0.84(95 \% \mathrm{CI}, 0.76-0.91 ; \mathrm{p}<0.0001)$ for BAC. (Fig. 1) Both the curves were statistically significant. The cutoff values established were 20.4 cells $/ \mu 1$ with sensitivity of $81.1 \%$, specificity $82.1 \%$ and Youden Index was 63.3 . For BAC counts, cut off of 143.2 cells $/ \mu 1$ was established with sensitivity of $81.1 \%$, specificity of $79.2 \%$ and Youden Index was 60.3.

Flow cytometry analysis of bacterial cells showed high sensitivity $(81.1 \%)$ as well as high negative predictive value $(93 \%)$. WBC counts showed high negative predictive value $(93 \%)$ and specificity of $81.5 \%$. Analysis of nitrites displayed $97 \%$ specificity and $81 \%$ negative predictive value while leucocyte esterase showed $86.9 \%$ specificity and $92.4 \%$ negative predictive value.

The parameters included in the study were evaluated in a group of 2 or more to evaluate the performance of the 
combination. Nitrites show highest specificity of $97 \%$ when evaluated individually. WBC and BAC counts both showed the $81.1 \%$ sensitivity, individually. Sensitivity was not increased in any combination while specificity increased to $100 \%$ if two or more values were combined with nitrites.

\section{Discussion}

As urinary tract infections are one of the most prevalent bacterial infections, detection of the same should be done in the least time possible. Analysis of the urine is the screening test performed to detect UTI and help guide the clinicians in formulating a treatment plan. It is

Table I: Analysis of multiple parameters for detection of UTI.

\begin{tabular}{|l|l|l|l|l|}
\hline & Sensitivity & Specificity & PPV $^{1}$ & NPV $^{2}$ \\
\hline Nit $^{3}$ & 0.283019 & 0.970238 & 0.75 & 0.810945 \\
\hline WBC $^{4}{ }^{5}$ & 0.773585 & 0.869048 & 0.650794 & 0.924051 \\
\hline BAC $^{6}$ & 0.811321 & 0.815476 & 0.581081 & 0.931973 \\
\hline Leu and Nit & 0.811321 & 0.791667 & 0.551282 & 0.93007 \\
\hline Leu and WBC & 0.226415 & 1 & 1 & 0.803828 \\
\hline Leu and BAC & 0.754717 & 0.880952 & 0.666667 & 0.919255 \\
\hline Nit and WBC & 0.679245 & 0.928571 & 0.75 & 0.901734 \\
\hline Nit and BAC & 0.245283 & 1 & 1 & 0.807692 \\
\hline WBC and BAC & 0.264151 & 1 & 1 & 0.811594 \\
\hline Leu, Nit and WBC & 0.716981 & 0.89881 & 0.690909 & 0.909639 \\
\hline Leu, Nit and BAC & 0.226415 & 1 & 1 & 0.803828 \\
\hline Leu, WBC and BAC & 0.226415 & 1 & 1 & 0.803828 \\
\hline Nit, WBC and BAC & 0.660377 & 0.934524 & 0.76087 & 0.897143 \\
\hline Leu, Nit, WBC and BAC & 0.245283 & 1 & 1 & 0.807692 \\
\hline
\end{tabular}

1 PPV: Positive Predictive Value

2 NPV: Negative Predictive Value

3 Nit: Nitrites

$4 \quad$ Leu: Leucocyte Esterase

5 WBC: White Blood Cells

6 BAC: Bacterial Counts

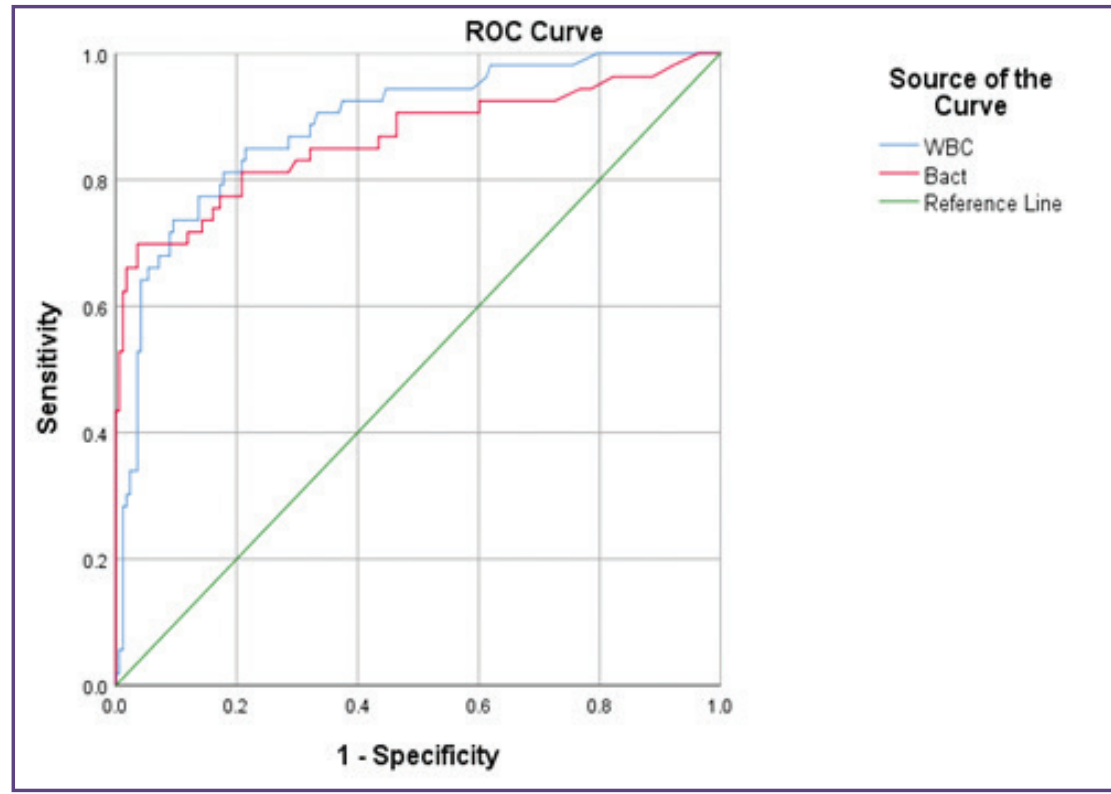

Fig. 1: Receiver Operating Curve (ROC) for WBC \& BAC. 


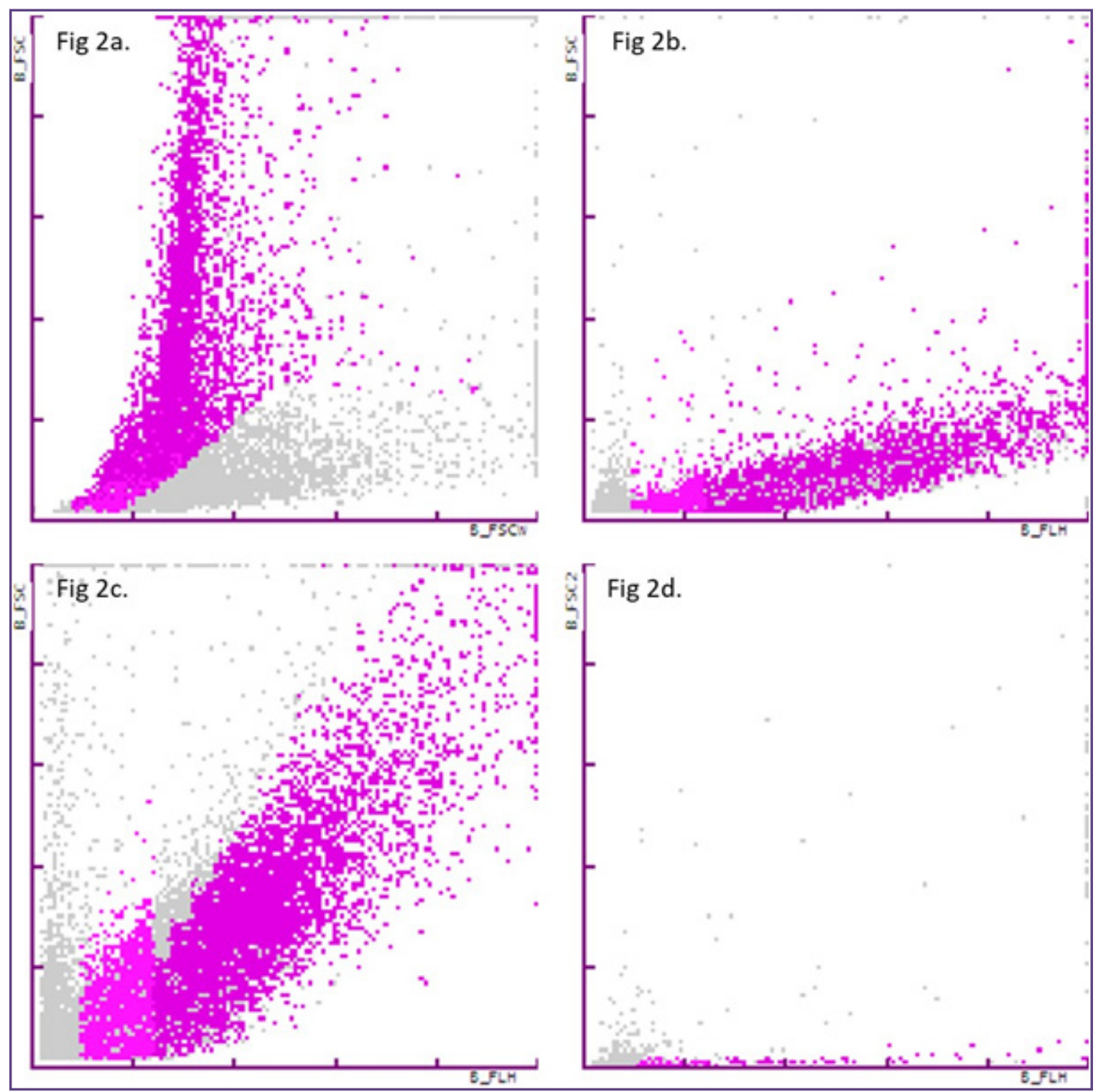

Fig. 2: Bacterial scatterplot patterns. a. Gram positive $\left(>30^{\circ}\right)$; b. Gram negative $\left(<30^{\circ}\right)$; c. Mixed infection; d. negative for bacteria [B_FSC: Bacteria forward scatter, B_FLH: Bacteria fluorescent light intensity].

a high volume, labor and time intensive test, which adds workload and cost for the laboratory. The gold standard for confirming UTI is by performing a culture of urine and it takes a minimum of $18-48$ hours to give a definite result. It is an added cost towards diagnosis of UTI and might not be always available in all clinical settings. ${ }^{[12]}$ The aim of the study was to determine parameters that could be used to detect with high certainty the cases which would turn out to be culture positive so that empirical treatment could be initiated without waiting for the culture reports.

The present study had more male subjects (133) as compared to females (103). This could be due to the fact that the study was conducted in a super specialty hospital with the Urology department which receives more referred male subjects as compared to female subjects. UTI was more prevalent in females in the current study. This is consistent with most of the studies conducted stating UTI is more prevalent among sexually active women. Numerous factors have been identified that can increase susceptibility to UTI which includes ABO blood-group antigens, congenital abnormalities, presence of urinary obstruction, previous history of UTI, use of diaphragms and estrogen deficiency. ${ }^{[17]}$

WBC and BAC counts are the most useful predictors for screening for UTI. There was a significant difference 
between the mean $\mathrm{WBC}$ and $\mathrm{BAC}$ counts of culture positive and negative samples, highlighting the fact that elevated levels of both could be used as a predictor of UTI. The high values in the ROC curve of both WBC and BAC counts establish that UX-2000 can distinguish between culture positive and culture negative cases. The difference between the curves of WBC and BAC is not much as to use one parameter more favorably.

The cutoff value of WBC of 20.4 cells/ $\mu$ l gave high sensitivity and specificity with high NPV and low false positive rate. Study by Santos et al came to the same conclusion; in their study at a cutoff of 20 cells/ $\mu 1$ gave sensitivity of $83.3 \%$ and specificity of $76.3 \%{ }^{[18]}$ However, it was lower as compared to other studies. ${ }^{[19-21]}$ For BAC counts, the cutoff was taken as 143.2 cells/ $\mu$ l with sensitivity of $81.1 \%$, specificity of $79.2 \%$ and $93 \%$ negative predictive value. At this cutoff, it detected 133 out of 143 negative cases correctly. This could be used to reduce the need for cultures by detecting true negative cases. De Rosa et al came to the same conclusion, with a cutoff of 170 cells/ $\mu$ l gave specificity of $76.5 \%$ and NPV of $99.5 \%{ }^{[20]}$ Though, the cutoff chosen was lower than other studies in literature. ${ }^{[21-23]}$ The cutoff values, for $\mathrm{WBC}$ and BAC, with high sensitivity (>95\%) was not selected, as it gave low specificity (17.8\% for BAC and $38.7 \%$ for $\mathrm{WBC}$ ).

Leucocyte esterase and nitrites are proven to have high specificity in various studies. ${ }^{\left[{ }^{[8}\right.}$ In this study, we came to the same conclusion. Individually, nitrite has the highest specificity of $97 \%$ with $75 \%$ positive predictive value and $81 \%$ negative predictive value. But when nitrite was combined with 2 or more parameters, the specificity increased to $100 \%$ and positive predictive value to $85.7 \%$. Leucocyte esterase showed high specificity and negative predictive value. However, leucocyte esterase did not show an increase in specificity when combined with other parameters. (Table 1)

Another observation in the current study was the distribution of bacteria on the scattergrams. The gram negative bacteria were seen to be distributed below $30^{\circ}$, whereas the gram positive bacteria were observed above $30^{\circ}$. This observation could guide in the establishment of a better treatment protocol, choosing more appropriate initial empirical antibiotics as well as a reduction in antimicrobial resistance. Another pattern observed was "wide distribution pattern", which signals the presence of mixed bacterial infection and needs correct identification via bacterial culture. (Fig. 2) A similar observation was noted in a study conducted in the Philippines but was conducted on Sysmex UF-1000i analyzer. ${ }^{[13]}$

\section{Conclusion}

Laboratory specific cutoff values should be established depending upon the patient population in addition to the diagnostic criteria taken and monitored regularly. It will aid in correctly screening out a majority of negative samples, thereby reducing unnecessary antibiotic prescriptions. Flow cytometry-based urine analyzers also reduces the turnaround time for the detection of negative samples by reducing the need for culture. In our opinion, WBC and $\mathrm{BAC}$ counts together could be used as a predictor of UTI while nitrite and leucocyte esterase could help identify negative cases. WBC, BAC, Leu and Nit have good predictive value and help reduce the workload of the laboratory by distinguishing between positive and negative urinary tract infections. In the end, we emphasize on the need for optimization of laboratory standards while increasing the quality of service provided at the same time for better patient care and upgradation of laboratory services.

\section{Abbreviations}

UTI: Urinary tract infection, WBC: White Blood Cells, RBC: Red Blood Cells, CHM: Cytochemistry, FCM: flow cytometry, BAC: Bacterial counts, Leu: Leucocyte esterase, Nit: Nitrites, AUC: Area under the curve

\section{Conflict of Interests}

The authors declare no conflict of interests.

\section{References}

1. Ahmed MA, Shukla GS, Bajaj HK. Incidence of urinary tract infections and determination of their susceptibility to antibiotics among pregnant women. International Journal of Cell Science and Biotechnology. 2016;5(2016):12-6.

2. Stamm WE, Norrby SR. Urinary Tract Infections: Disease Panorama and Challenges. The Journal of Infectious Diseases. 2001;183(Supplement_1):S1-S4. doi: 10.1086/318850.

3. Akortha EE, Ibadin OK. Incidence and antibiotic susceptibility pattern of Staphylococcus aureus among patients with urinary tract infection (UTI) in UBTH Benin City, Nigeria. African Journal of Biotechnology. 2008;7(11):1637-40.

4. Amin M, Mehdinejad M, Pourdangchi Z. Study of bacteria isolated from urinary tract infections and determination of their susceptibility to antibiotics. Jundishapur Journal of Microbiology. 2009;2(3):118-23.

5. Bent S, Nallamothu BK, Simel DL, Fihn SD, Saint S. Does this woman have an acute uncomplicated urinary tract infection? JAMA. 2002;287(20):2701-10. doi: 10.1001/ jama.287.20.2701.

6. Daoud Z, Afif C. Escherichia coli Isolated from Urinary Tract Infections of Lebanese Patients between 2000 and 2009: Epidemiology and Profiles of Resistance. 
Chemotherapy Research and Practice. 2011;2011. doi: 10.1155/2011/218431.

7. Ben-Ezra J, Bork L, McPherson RA. Evaluation of the Sysmex UF-100 automated urinalysis analyzer. Clinical Chemistry. 1998;44(1):92-5.

8. John AS, Boyd JC, Lowes AJ, Price CP. The use of urinary dipstick tests to exclude urinary tract infection. Am J Clin Pathol. 2006;126:428-36.

9. Devillé WLJM, Yzermans JC, van Duijn NP, Bezemer PD, van der Windt DAWM, Bouter LM. The urine dipstick test useful to rule out infections. A meta-analysis of the accuracy. BMC Urology. 2004;4:4. doi: 10.1186/1471-2490-4-4. PubMed PMID: PMC434513.

10. Simerville JA, Maxted WC, Phira JJ. Urinalysis: A comprehensive review. Am Fam Physician. 2005 Mar 15;71(6):1153-62.

11. Schmiemann G, Kniehl E, Gebhardt K, Matejczyk MM, Hummers-Pradier E. The Diagnosis of Urinary Tract Infection: A Systematic Review. Deutsches Ärzteblatt International. 2010;107(21):361-7. doi: 10.3238/ arztebl.2010.0361. PubMed PMID: PMC2883276.

12. Williams GJ, Macaskill P, Chan SF, Turner RM, Hodson E, Craig JC. Absolute and relative accuracy of rapid urine tests for urinary tract infection in children: a meta-analysis. The Lancet Infectious Diseases. 2010;10:240-50. doi: 10.1016/ S1473-3099(10)70031-1.

13. Agpaoa VV, Mendoza JB, Fernandez AJM, Veloso JD, Bhatnagar S. Predict urinary tract infection and to estimate causative bacterial class in Philippine subspecialty hospital. J Nephrol Ther. 2015;5(2):194. doi: 10.4172/21610959.1000194 .

14. Fully automated integrated urine analyzer UX-2000. Instructions for use. Kobe, Japan: Sysmex Corporation; 2015.

15. Khejonnit V, Pratumvinit B, Reesukumal K, Meepanya S, Pattanavin C, Wongkrajang P. Optimal criteria for microscopic review of urinalysis following use of automated urine analyzer. Clinica Chimica Acta. 2015;439:1-4. doi: https://doi.org/10.1016/j.cca.2014.09.027.

16. Clinical and Laboratory Standards Institute, Performance standards for antimicrobial susceptibility testing. CLSI document M100-S15. Wayne, PA, USA: Clinical and Laboratory Standards Institute; 15th International supplement, 2005.

17. Foxman B. Epidemiology of urinary tract infections: incidence, morbidity, and economic costs. The American Journal of Medicine. 2002;113(1):5-13. doi: 10.1016/ S0002-9343(02)01054-9.

18. Santos JCd, Weber LP, Perez LRR. Evaluation of urinalysis parameters to predict urinary-tract infection. The Brazilian Journal of Infectious Diseases. 2007;11(5):479-81.

19. Manoni F, Fornasiero L, Ercolin M, Tinello A, Ferrian M, Hoffer P, et al. Cutoff values for bacteria and leukocytes for urine flow cytometer Sysmex UF-1000i in urinary tract infections. Diagnostic Microbiology and Infectious Disease.65(2):103-7. doi: 10.1016/j. diagmicrobio.2009.06.003.

20. De Rosa R, Grosso S, Bruschetta G, Avolio M, Stano P, Modolo ML, et al. Evaluation of the Sysmex UF1000i flow cytometer for ruling out bacterial urinary tract infection. Clinica Chimica Acta. 2010;411(15):1137-42..

21. Broeren MAC, Bahçeci S, Vader HL, Arents NLA. Screening for Urinary Tract Infection with the Sysmex UF-1000i Urine Flow Cytometer. Journal of Clinical Microbiology. 2011;49(3):1025-9.

22. Kim SY, Kim YJ, Lee SM, Hwang SH, Kim HH, Son HC, et al. Evaluation of the Sysmex UF-100 Urine Cell Analyzer as a Screening Test to Reduce the Need for Urine Cultures for Community-Acquired Urinary Tract Infection. American Journal of Clinical Pathology. 2007;128(6):922-5. doi: 10.1309/4606EC29U50DVAFY.

23. Yang C-C, Chang S-J, Yang SS-D, Lin C-D, Peng C-H. Rapid diagnosis of uncomplicated urinary tract infection with laser flow cytometry. Urological Science. 2016;27(3):135-9. doi: https://doi.org/10.1016/j.urols.2016.09.001.

*Corresponding author:

Dr. Lalit Kumar, Department of Pathology, Rajiv Gandhi Super Specaility Hospital, Tahirpur, Delhi 110093, India

Phone: +91 9999744584

Email: drlkumar@gmail.com

Financial or other Competing Interests: None. 